LA-UR- $10-02891$

Approved for public release;

distribution is unlimited.

Title:

Stable and responsive fluorescent carbon nanotube silica gels

Author(s): $\quad$ Gautam Gupta, Juan G. Duque, S. K. Doorn, and Andrew M. Dattelbaum

Intended for:

Materials Research Society Spring 2010 meeting

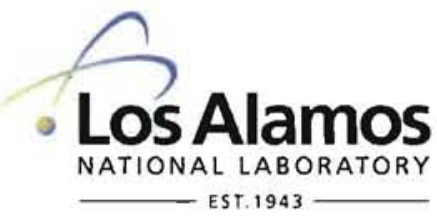

Los Alamos National Laboratory, an affirmative action/equal opportunity employer, is operated by the Los Alamos National Security, LLC for the National Nuclear Security Administration of the U.S. Department of Energy under contract DE-AC52-06NA25396. By acceptance of this article, the publisher recognizes that the U.S. Government retains a nonexclusive, royalty-free license to publish or reproduce the published form of this contribution, or to allow others to do so, for U.S. Government purposes. Los Alamos National Laboratory requests that the publisher identify this article as work performed under the auspices of the U.S. Department of Energy. Los Alamos National Laboratory strongly supports academic freedom and a researcher's right to publish; as an institution, however, the Laboratory does not endorse the viewpoint of a publication or guarantee its technical correctness.

Form $836(7 / 06)$ 


\title{
Stable and Responsive Fluorescent Carbon Nanotube Silica Gels
}

\author{
Gautam Gupta $^{1, *}$, Juan G. Duque ${ }^{2, *}$, Stephen K. Doorn ${ }^{1}$, and Andrew M. Dattelbaum ${ }^{1}$ \\ 'Center for Integrated Nanotechnologies, MS K771, Los Alamos National \\ Laboratory, Los Alamos, NM 87545 \\ ${ }^{2}$ Chemistry Division, Los Alamos National Laboratory, Los Alamos, NM 87545 \\ ${ }^{*}$ Authors contributed equally to work
}

\begin{abstract}
Here we report a general route to prepare silica nanocomposite gels doped with fluorescent single walled carbon nanotubes (SWNT). We show that tetramethylorthosilicate (TMOS) vapors can be used to gel an aqueous suspension of surfactant-wrapped SWNT while maintaining fluorescence from the semiconducting nanotubes. The vapor phase silica process is performed at room temperature and is simple, reproducible, relatively quick, and requires no dilution of SWNT dispersions. However, exposure of aqueous SWNT suspensions to TMOS vapors resulted in an acidification of the suspension prior to gelation that caused a decrease in the emission signal from sodium dodecylsulfate (SDS) wrapped SWNT. We also show that although the SWNT are encapsulated in silica the emission signal from the encapsulated SWNT may be attenuated by exposing the nanocomposites to small aromatic molecules known to mitigate SWNT emission. These results demonstrate a new route for the preparation of highly luminescent SWNT/silica composite materials that are potentially useful for future sensing applications.
\end{abstract}

\section{INTRODUCTION}

Single walled carbon nanotubes (SWNT) possess unique mechanical, electrical and luminescence properties due to their nanoscale structure based on a cylindrical sheet of graphene. (1-3) As such, carbon nanotubes show a promising future in the fields of sensing, optics, nanotechnology, electronics, and materials science. Recently, we have been particularly focused on exploiting the near-infrared (NIR) emission signal arising from isolated semiconducting SWNT for sensing applications. $(4,5)$ Isolation of SWNT can be readily accomplished by suspending SWNT in aqueous solution via addition of surfactants.(6, 7) In order to fully harness the unique NIR properties of SWNT, there is a need to develop nanocomposites that readily incorporate isolated nanotubes. Silica is an ideal material for encapsulation of fluorescent SWNT, as it is generally inert, optically transparent, and permeable.(5) Here we used a vapor diffusion method to prepare silica nanocomposites containing fluorescent SDS-wrapped SWNT. The SWNT/silica nanocomposites prepared in this way retain over $50 \%$ of the initial emission signal intensity observed from SWNT prior to encapsulation. In addition, the SWNT luminescence signal was shown to be sensitive to the addition of small aromatic compounds, which demonstrates the possibility of future sensing applications with these materials. 


\section{EXPERIMENTAL}

SWNT were synthesized by high-pressure decomposition of carbon monoxide (HiPco). $(6,8)$ The SWNT dispersion was prepared at $1 \mathrm{wt} \%$ sodium dodecylsulfate (SDS) in $\mathrm{D}_{2} \mathrm{O}$ using standard suspension procedures.(7) A SWNT aqueous dispersion $(\sim 1 \mathrm{ml})$ was placed in a Petri dish along with a Teflon-coated cap containing TMOS $(0.5 \mathrm{ml}$, Aldrich, $99 \%)$ in a closed container for at least 1-2 hours at room temperature. After the desired exposure time, the SWNT suspension was aged at room temperature to form a gel. Fluorescence and FT-IR spectroscopy techniques were used to characterize the resulting gels. An aromatic molecule, 4amino-1,1-azobenzene-3,4-disulphonic acid (AB, Aldrich; $1 \mathrm{M}$ (aq)) was used to probe the sensitivity of SWNT emission in both the aqueous suspension and silica gel samples.

\section{RESULTS AND DISCUSSION}

\section{Synthesis of gels}

A schematic of the chemical vapor deposition process used to prepare SWNT/silica nanocomposites is shown in Fig. 1A. Open containers of tetramethylorthosilicate (TMOS) and an aqueous dispersion of SDS-wrapped SWNT were placed inside a larger vessel that was sealed in air and kept at room temperature.(9) Due to the volatile nature of TMOS at room temperature, the precursor quickly fills the atmosphere in the sealed vessel. When the vapors reach the aqueous solution/air interface they hydrolyze to form silicilic acid and methanol according to equation 1.

(A)

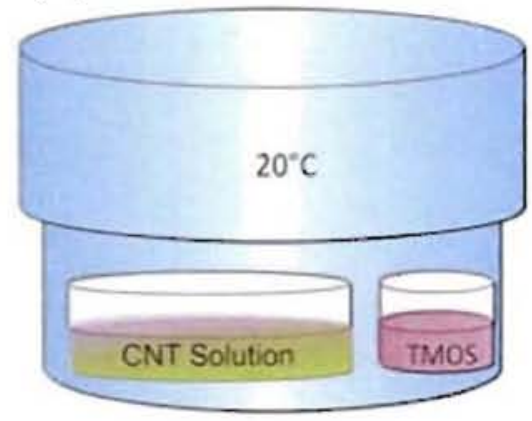

(B)

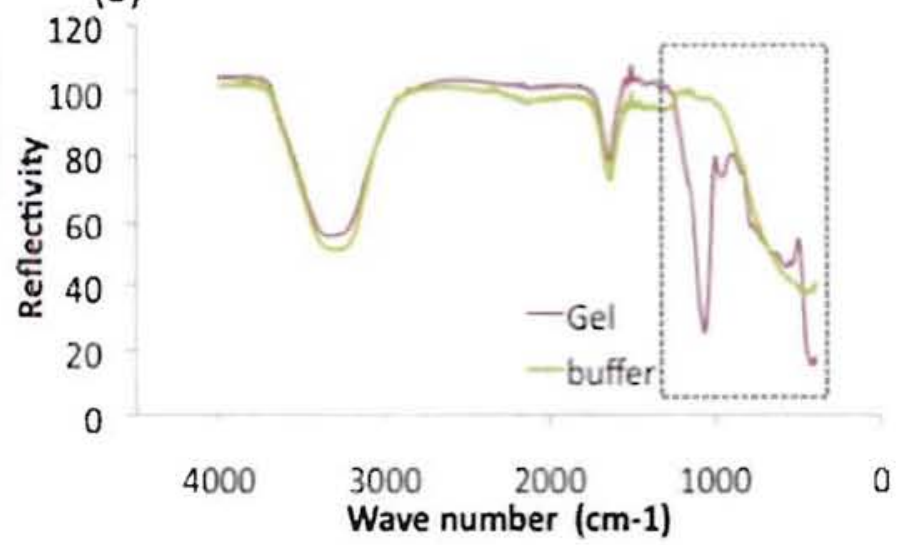

Figure 1. (A) Schematic of the vapor transfer process used to prepare SDS/SWNT/silica gel nanocomposites. (B) FT-IR spectra of $10 \mathrm{mM}$ sodium phosphate buffer (i) before exposure (green) and (ii) after exposure (pink) to TMOS.

Further condensation leads to formation of Si-O-Si linkages resulting in a homogeneous silica gel (equation 2).

$$
\begin{aligned}
& \mathrm{Si}\left(\mathrm{OCH}_{3}\right)_{4}+4 \mathrm{H}_{2} \mathrm{O} \rightarrow \mathrm{Si}(\mathrm{OH})_{4}+4 \mathrm{CH}_{3} \mathrm{OH} \\
& \mathrm{Si}(\mathrm{OH})_{4} \rightarrow \mathrm{SiO}_{2}+2 \mathrm{H}_{2} \mathrm{O}
\end{aligned}
$$


The slow rate of transfer of TMOS precursor molecules into the SWNT aqueous suspension leads to minimal methanol presence in the aqueous solution at any given time. This is advantageous because methanol can disrupt the SDS/SWNT assembly leading to aggregation of SWNT and loss of emission. To demonstrate the lack of methanol in the as prepared gels, an FT-IR spectrum of an aqueous solution before and after exposure to TMOS vapor is shown in Fig. 1B. Notice that there are no significant features around $2900 \mathrm{~cm}^{-1}$ where one would expect $\mathrm{C}$-H modes to be present due to methanol or incomplete condensation of TMOS. In addition, both spectra have a broad absorption band between 4000 and $3000 \mathrm{~cm}^{-1}$ that corresponds to the fundamental stretching vibrations of different types of hydroxyl groups $(\mathrm{H}-$ $\mathrm{OH}$ and $\mathrm{Si}-\mathrm{OH}$ in our case). The spectrum taken after exposure to TMOS vapors also shows new bands at 1062, 964, 810 and $445 \mathrm{~cm}^{-1}$ (highlighted by the box in Fig. 1B). Bands at similar wave numbers in the spectra of crystalline and amorphous $\mathrm{SiO}_{2}$ have been assigned to characteristic vibrations of Si-O-Si bridges cross-linking the silicate network.

\section{Fluorescence from SWNT Nanocomposites}

Near-infrared emission observed from SDS-wrapped SWNT in solution and upon encapsulation in a silica gel is shown in Fig. 2.

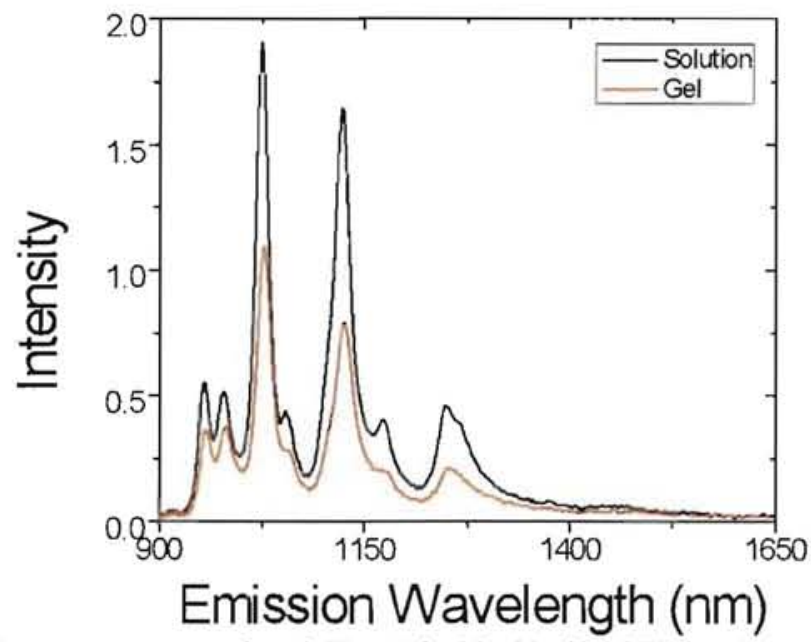

Figure 2. (A) SDS/SWNT aqueous suspension ( $\mathrm{pH}=7$ in black), (B) Silica gel prepared from a suspension of SDS/SWNT ( $\mathrm{pH} \sim 5.5$ after exposure to TMOS vapor in red). Fluorescence spectra collected using an excitation wavelength of $640 \mathrm{~nm}$.

Each emission peak is due to a different chirality of SWNT. Notice that all of the emission features observed from the SWNT dispersions were maintained in the SWNT/silica gels, although there was an overall decrease in fluorescence intensity upon encapsulation. In particular, the larger diameter tubes that emit at longer wavelengths decreased to a much greater extent. These changes in emission behavior are consistent with previous results observed upon acidification of surfactant-wrapped SWNT aqueous dispersions.(10) In fact, a significant drop in $\mathrm{pH}$ was measured by a $\mathrm{pH}$ meter before and after exposure to TMOS vapor. The initial SWNT suspension had a $\mathrm{pH}$ of $\sim 7$, while after exposure to TMOS the $\mathrm{pH}$ dropped to 5.5. Interactions 
between protons in solution and SWNT are known to quench totoluminescence signal from SWNT.(8)

\section{Bleaching of SWNT emission by Aromatic Molecules}

The SDS-wrapped SWNT in solution and encapsulatedica were exposed to small azobenzene-type (AB) molecules that have previously been sho bleach the SWNT photoluminescence signal in suspension and silica. $(5,11)$ Fluonce spectra were recorded before and after $4 \mathrm{hrs}$ of $\mathrm{AB}$ exposure. This time was sufficientromplete diffusion of $\mathrm{AB}$ into the silica gels containing SWNT, and also to achieve an emissidy state. As seen in Fig. 3, the SDS/SWNT suspension and SWNT/silica gels respond sim with virtually complete bleaching upon addition of $A B$. These results suggest that the sulation of the SWNT in silica does not inhibit the accessibility of small molecules to the nanotubes.

(A)

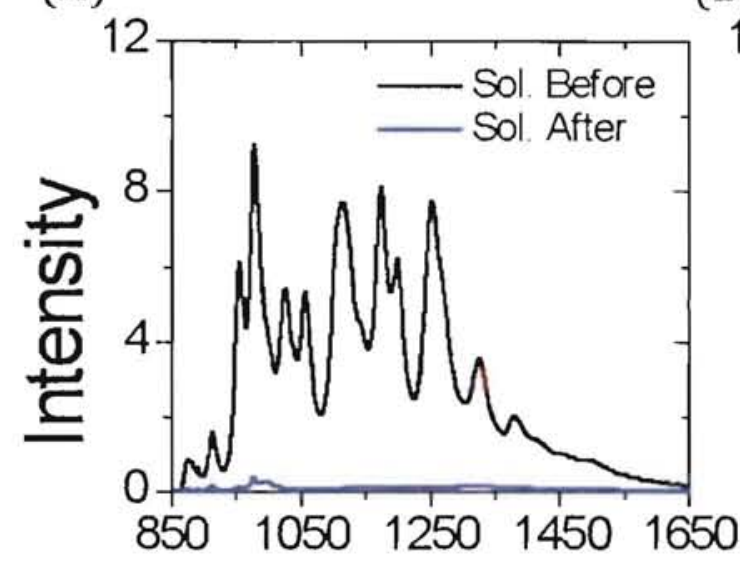

(B)

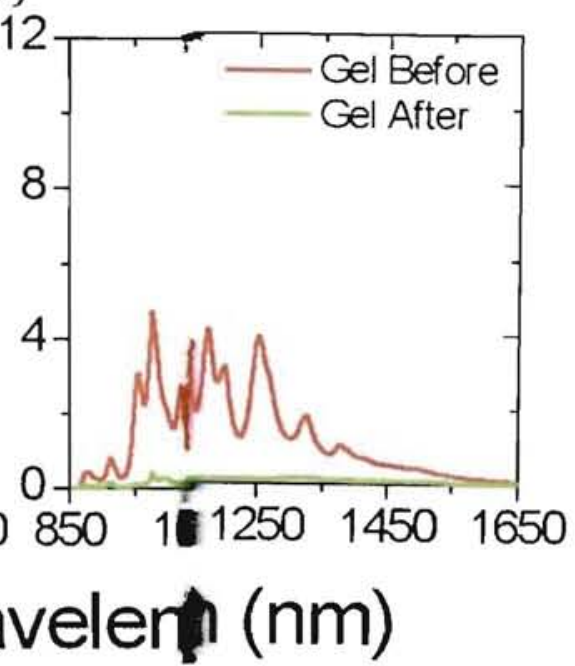

Figure 3. Fluorescence spectra for SWNT in solution and silica gels upoure to azobenzene-type molecules. (A) SDS/SWNT (suspension) before (black) and after AB exp hrs (blue). (B) SDS/SWNT encapsulated in silica before (red) and after $\mathrm{AB}$ exposure, $4 \mathrm{hrs}$ (green). Fence spectra collected using an excitation wavelength of $785 \mathrm{~nm}$.

\section{CONCLUSIONS}

We have successfully shown the immobilization of surf-wrappc... SWNT in silica gels using a vapor phase process. The photoluminescence intenfom encapsulated SWNT was maintained after formation of the silica gel. We also show emission from SWNT encapsulated in silica may still be mitigated by exposure to smatic molecules. These results demonstrate a new route for the preparation of highly lucent SWNT/silica composite materials potentially useful for future sensing applic 


\section{ACKNOWLEDGEMENTS}

Funding for this work was provided by the DOE-BES, Office of Science. The authors thank Dr. Gabriel P. Lopez (Duke University) for helpful discussions on synthesizing stable silica gels. JGD would also like to thank the LANL-LDRD Director's Postdoc Fellowship Program for support.

\section{REFERENCES}

1. R. H. Baughman, A. A. Zakhidov, W. A. de Heer, Science 297, 787 (August 2, 2002, 2002).

2. J. Lefebvre, S. Maruyama, P. Finnie, in Carbon Nanotubes. (Springer-Verlag Berlin, Berlin, 2008), vol. 111, pp. 287-319.

3. H. Dai, Surface Science 500, 218 (2002).

4. B. C. Satishkumar et al., Nature Nanotechnology 2, 560 (Sep, 2007).

5. B. C. Satishkumar, S. K. Doorn, G. A. Baker, A. M. Dattellbaum, ACS Nano 2, 2283 (Nov, 2008).

6. S. M. Bachilo et al., Journal of the American Chemical Society 125, 11186 (2003).

7. M. J. O'Connell et al., Science 297, 593 (July 26, 2002, 2002).

8. P. Avouris, M. Freitag, V. Perebeinos, in Carbon Nanotubes. (Springer-Verlag Berlin, Berlin, 2008), vol. 111, pp. 423-454.

9. G. Gupta et al., Langmuir 25, 13322 (2009).

10. J. G. Duque et al., Journal of the American Chemical Society 130, 2626 (2008).

11. M. J. O'Connell, E. E. Eibergen, S. K. Doorn, Nature Materials 4, 412 (May, 2005). 